\title{
Hypothesis: What is the Best We Can Do with Hydroxychloroquine for COVID-19?
}

This article was published in the following Dove Press journal: Clinical Epidemiology

\author{
Shuncong Wang ${ }^{1, *}$ \\ Stefaan Mulier ${ }^{1,2, *}$ \\ Charles Jonscher iD $^{3}$ \\ Sheng $\mathrm{Ye}^{4}$ \\ Lei Chen' \\ Yuanbo Feng' \\ Yue $\mathrm{Li}^{5}$ \\ Yicheng $\mathrm{Ni}$ (iD) \\ 'Campus Gasthuisberg, Group of \\ Biomedical Sciences, KU, Leuven, \\ Belgium; ${ }^{2}$ Department of Surgery, Delta \\ Hospital, Brussels, Belgium; ${ }^{3}$ Institute for \\ Health Metrics \& Evaluation, University of \\ Washington, Seattle, WA, USA; ${ }^{4}$ Primary \\ Care NJ, Englewood, NJ, USA; ${ }^{5}$ Shanghai \\ Key Laboratory of Molecular Imaging, \\ Shanghai University of Medicine and \\ Health Sciences, Shanghai 201318, \\ People's Republic of China \\ *These authors contributed equally to \\ this work
}

\begin{abstract}
There are widespread anecdotal reports of seemingly successful treatment among the early (three to seven days from symptoms) stage coronavirus disease 2019 (COVID-19) patients with the drug hydroxychloroquine (HCQ), and randomized placebo-controlled trials of HCQ in outpatient settings are underway. In this note, we (1) report observational evidence and present scientific reasoning as to why early treatment with HCQ may succeed while treatment later in the disease progression is likely to fail and (2) hypothesize a public health regime under which HCQ may be used to mitigate the impact of the current pandemic.
\end{abstract}

Keywords: COVID-19, pandemic, early treatment, hydroxychloroquine, lockdown

\section{Introduction}

The current coronavirus disease 2019 (COVID-19) outbreak caused by severe acute respiratory syndrome coronavirus 2 (SARS-CoV-2) has swept the world since late $2019,{ }^{1}$ with over 36.8 million confirmed patients and nearly 1.1 million deaths to date, ${ }^{2}$ leading to unprecedented societal costs and a global economic crisis. Now, countries are confronting the dilemma of how to resume normalcy after lockdowns while still containing the current COVID-19 pandemic and its possible subsequent resurgences. ${ }^{3,4}$

Few medications for COVID-19 have been approved by the US Food and Drug Administration (FDA) and other authorities. ${ }^{5}$ Clinical management relies mainly on spontaneous recovery by the patient, symptomatic treatment and implementation of supportive measures. Due to the urgent nature of this viral pandemic, physicians can, under emergency use authorizations (EUA) and emergency investigational new drug (EIND) rules, access and prescribe existing medicines approved or licensed for other indications to COVID-19 patients for compassionate, expanded or off-label use.

Currently, for the treatment of COVID-19, there are thousands of clinical trials around the globe on numerous new investigational agents or drugs approved already for other indications; ${ }^{6}$ among these are chloroquine (CQ) and its derivative hydroxychloroquine (HCQ, widely marketed as Plaquenil®), used for decades as antimalaria drugs and found to have broad-spectrum antiviral potential, ${ }^{7}$ with HCQ being preferred to CQ for its better efficacy and safety. ${ }^{8} \mathrm{HCQ}$ has also been approved as an immunomodulatory agent for the long-term treatment of chronic rheumatic diseases such as systemic lupus erythematosus and rheumatoid arthritis. ${ }^{9}$ However, HCQ and CQ are controversial in terms of their efficacy and safety for clinical control of COVID-19. ${ }^{10,11}$ A large-scale registry analysis of data from 671 hospitals on six continents recently 
reported no observed benefit in patients, instead an increased risk in-hospital mortality, associated with treatment of COVID-19 patients with either HCQ and CQ (with or without a macrolide); however, this study was subsequently withdrawn by three of its four authors. ${ }^{12}$ In a retrospective study of 1438 hospitalized patients, the treatment with HCQ, azithromycin, or both, compared with neither treatment, was not significantly associated with differences in in-hospital mortality. $^{13}$

\section{Potential Efficacy and Its Possible Mechanisms}

Despite controversies, accumulating evidence has been demonstrating the potential anti-COVID-19 activity of both HCQ and CQ, on the basis of observational studies, randomized controlled clinical trials and basic studies. ${ }^{5,8-12,14-16}$ Risch reviews in detail the experiences in the USA and other countries of administering HCQ, with or without an antibiotic, to early stage COVID-19 patients, concluding that the observational evidence of efficacy is very strong; ${ }^{17}$ formal clinical trials in an outpatient setting have now begun, with the first results expected in September, 2020.

HCQ and CQ may exert antiviral activities in the following possible ways: ${ }^{9,18}$ (1) reduce the terminal glycosylation of angiotensin-converting enzyme 2 (ACE2) receptor on the surface of cells, thus interfering with the binding of SARS-CoV-2 to the ACE2 receptor; (2) increase the $\mathrm{pH}$ of lysosomes and endosomes to prevent the fusion process of the virus with host cells and subsequent virus replication; (3) prevent antigen processing and major histocompatibility complex class II-mediated autoantigen presentation to $\mathrm{T}$ cells, which reduces $\mathrm{T}$ cell activation, differentiation and expression of co-stimulatory proteins and cytokines (eg, IL-1, IL-6 and TNF- $\alpha$ ) produced by T cells and B cells; (4) disrupt the interaction of DNA/RNA with toll-like receptors (TLRs) and the nucleic acid sensor cyclic GMP-AMP (cGAMP) synthase (cGAS) and therefore the transcription of pro-inflammatory genes cannot be stimulated; ${ }^{19}$ and (5) serve as an ionophore for zinc, an inhibitor for RNA dependent RNA polymerase and furin that cleaves the spike of SARS-CoV-2 and thus promotes viral infection. ${ }^{20-23}$ A retrospective clinical study demonstrated the clinical benefit of combinatory use of HCQ and zinc, ${ }^{24}$ which should be further validated by randomized controlled trials.
Now, it is known that COVID-19 is primarily a respiratory disease but also an illness that can affect multiple organs. ${ }^{25,26}$ When the SARS-CoV-2 virus enters the human body, it breaks into cells with the help of two proteins, ACE2 and transmembrane protease serine 2 (TMPRSS2), which are expressed on the cell. There are enriched ACE2 and TMPRSS2 at olfactory epithelium of nasal mucosa that presents the first frontline per SARSCoV-2 invasion. ${ }^{27}$ In this sense, HCQ should be administered early to COVID-19 patients within the first week of symptoms onset to prevent the virus from infecting the deeper type 2 lung cells through the ACE2 receptors and thus to stop disease progression.

According to current COVID-19 guidelines, HCQ and CQ should only be used in clinical trials or emergency use programmes. $^{28,29}$ The previously reported unsatisfactory outcomes in severe stage hospitalized patients ${ }^{10-12,30}$ are understandable, because the disease has advanced to the deeper airway with the characteristic X-ray ground glass opacity and to multiple other organs, even without remaining SARS-CoV-2; and all severe pulmonary, renal, cardiovascular and cerebral complications come from late stage of disease pathologies with a high death rate.

However, the SARS-CoV-2 load is low during early onset before massive viral replications in the body, hence only a low dose of HCQ is needed, thus better safety, without additional need for azithromycin that is known to be synergistic with HCQ or CQ for cardiac toxicity. ${ }^{5,30,31}$ Therefore, both timing and dose appear crucial for the optimal use of HCQ in COVID-19 treatment. More recently, sequela such as fatigue, shortness of breath and headache are reported among patients three months after their recovery, even those with "mild" symptoms can suffer from COVID-19 for a long time. ${ }^{32}$ However, among our own over 200 cumulated early outpatient cases successfully managed with small doses of HCQ, none of them complained of similar prolonged sequela months after their recovery.

\section{Hypothetic Protocol for Implementing a Strategy to Contain the COVID-19 Pandemic Based on Administering HCQ Early in the Disease Onset}

Self-evidently, observational evidence such as that referenced in this paper is an insufficient basis on which to implement nationwide strategies and protocols for administration of HCQ to early stage COVID-19 patients; the 
success of the treatment remains a hypothesis. However, given the strong observational signals, it is not too early to start work on the design and planning of such strategies and protocols, in anticipation of possible (or likely in the authors' view) positive outcomes of the randomized controlled clinical trials. It is accepted by public health authorities and medical regulatory bodies that the very high number of continuing fatalities in the current pandemic calls for a speeding up of the normal timescales and processes for drug development and approval. ${ }^{17}$ Absence of evidence does not necessarily imply evidence of absence. Additionally, given the urgency of the pandemic, some flaws in the published studies may be acceptable, such as lack of randomization or blinding, retrospective design and adoption of an unvalidated surrogate of end point, etc.

It is known that infection-control strategies focused solely on symptomatic residents are not sufficient to prevent transmission, ${ }^{33}$ and conventional infection-control and public health strategies rely heavily on early detection of the disease to contain spread. ${ }^{34}$ In both cases, identification of laboratoryconfirmed individuals is essential. Given the presence of asymptomatic SARS-COV-2 carriers and presymptomatic
COVID-19 patients at incubation stage, containment of SARS-CoV-2 transmission would anyway be challenging ${ }^{33-}$ ${ }^{35}$ despite the extremely high costs for all preventive measures.

Given the rather cost-effective and safe outcomes from recent observational experiences with $\mathrm{HCQ}^{8,9,11,14,16,31}$ pending the outcomes of the ongoing clinical trials, we would propose, prepare now and implement promptly the following outline protocol for this and possibly subsequent outbreaks of COVID-19 (Figure 1).

1. By way of preparation, ensure the availability of sufficient quantities of the (easily prepared, small molecule) drug in oral doses, together with guidelines for prescription and use consistent with the regime outlined in this protocol. Proper provision, allocation and distribution of HCQ or CQ may help balance the treatment between COVID-19 patients and dermatological or rheumatic patients who are previously prescribed with these drugs. ${ }^{36}$

2. Additionally, make tests for the pathogens such as SARS-CoV-2 and/or their relevant serum antibody ready as early and as widely available as possible.

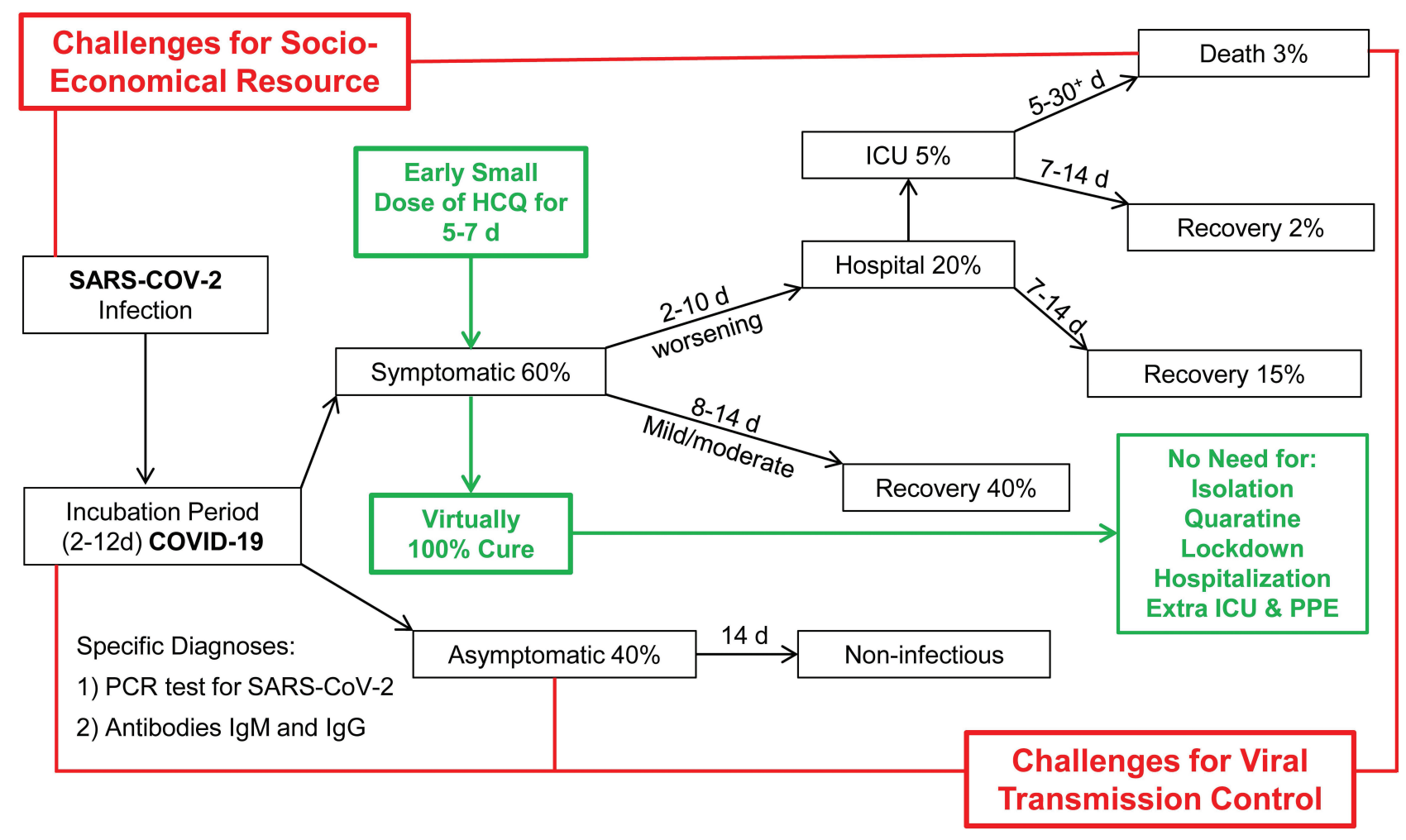

Figure I A hypothetic schematic regime for COVID-I9 pandemic control: estimated clinical scenarios (black), socioeconomic and epidemiological challenges (red), and a potentially simple solution (green).

Abbreviations: SARS-CoV-2, severe acute respiratory syndrome coronavirus 2; COVID-19, coronavirus disease 2019; PCR, polymerase chain reaction; IgM, immunoglobulin M; IgG, immunoglobulin G; HCQ, hydroxychloroquine; d, days; ICU, intensive care unit; PPE, personal protective equipment; +, or more. 
3. In positive-testing patients or in suspected cases without tests, administer HCQ at a dose to be finalized in light of the clinical trial results, but which we envision will be in the range of 100-200 mg, twice or thrice per day, for 5-10 days, a level which is likely to be highly efficacious because of patients' low viral load at this early stage and which can prevent patients from advancing to more severe stages.

4. Start interventions as quickly as possible, and ideally within three to seven days of symptom onset, well before the disease advances to the patients' lungs (pneumonia) or other visceral organs, which otherwise would be too late to achieve optimal medical and societal outcomes.

5. Antibiotics such as azithromycin can be combined especially when bacterial infections are observed; however in the absence of such observations, administration of HCQ would not be accompanied by azithromycin that may complicate the therapeutic regime with a known synergy for cardiac toxicity. ${ }^{5,30}$ When the patient is dosed with HCQ or CQ and azithromycin, electric cardiograph (ECG) is recommended to use for monitoring potential cardiac adverse effects such as QT prolongation. ${ }^{37,38}$

6. Repeat or perform the nucleic acid tests, antibody tests and/or T cell tests to follow-up the therapeutic efficacy and to monitor potential postinfection herd immunity. ${ }^{39}$ Concerning immunity of convalescent patients, the adopted immunity is likely mediated by SARS-CoV-2-specific $T$ cells, rather than antibody. ${ }^{40,41}$ More interestingly, people with mild or asymptomatic COVID-19 demonstrate T-cellmediated immunity, even if they have not tested positively for antibodies. ${ }^{42}$ Herd immunity thus achieved actively (ie, through therapy-mediation) would avoid the heavy mortality in the population, which would be required to achieve herd immunity passively in the current and possible future outbreaks of COVID-19. ${ }^{43}$

7. Societal regimes for protecting individuals against infection transmission, such quarantines, social distancing, city lockdowns, should not generally be needed under this protocol, but should continue to be applied mainly to those population cohorts, such as the elderly or those with certain pre-existing health conditions, who are particularly susceptible for SARS-CoV-2 infection.
In summary, we believe that, following confirmatory clinical studies, the early administration of HCQ, with an antibiotic or zinc where indicated, could become the front line of the global strategic response to this and later outbreaks of COVID-19, allowing a great reduction in the need for measures such as quarantines, isolations, social distancing, city lockdowns and in the need for hospitalizations. We have hypothesized a protocol for implementing this strategy, which we propose should be refined and prepared in parallel with, rather than delayed until the definitive outcomes of placebo controlled randomized clinical trials among early ( $<7$ days) onset COVID-19 patients.

\section{Author Contributions}

All authors made substantial contributions to conception and design, clinical observations, literature search and interpretation; taking part in drafting the article or revising it critically for important intellectual content; agreement to submit to the current journal; giving final approval of the version to be published; and agreeing to be accountable for all aspects of the work.

\section{Funding}

No funding is received for current study.

\section{Disclosure}

The authors report no conflicts of interest in this work.

\section{References}

1. Gates B. Responding to Covid-19 - a once-in-a-century pandemic? N Engl J Med. 2020;382(18):1677-1679. doi:10.1056/NEJMp2003762

2. COVID-19 CORONAVIRUS PANDEMIC. https://www.world ometers.info/coronavirus/. Accessed October 9, 2020.

3. Peto J, Alwan NA, Godfrey KM, et al. Universal weekly testing as the UK COVID-19 lockdown exit strategy. Lancet. 2020;395 (10234):1420-1421. doi:10.1016/S0140-6736(20)30936-3

4. Kissler SM, Tedijanto C, Goldstein E, Grad YH, Lipsitch M. Projecting the transmission dynamics of SARS-CoV-2 through the postpandemic period. Science. 2020;368(6493):860-868. doi:10.11 26/science.abb5793

5. Ahsan W, Javed S, Bratty MA, Alhazmi HA, Najmi A. Treatment of SARS-CoV-2: how far have we reached? Drug Discov Ther. 2020;14 (2):67-72. doi:10.5582/ddt.2020.03008

6. Clinical trials on Covid19. Available from: https://www.clinicaltrials. gov/ct $2 /$ results? cond $=$ Covid $19 \&$ term $=\&$ cntry $=\&$ state $=\&$ city $=\&$ dist $=$. Accessed October 9, 2020.

7. Savarino A, Di Trani L, Donatelli I, Cauda R, Cassone A. New insights into the antiviral effects of chloroquine. Lancet Infect Dis. 2006;6(2):67-69. doi:10.1016/S1473-3099(06)70361-9

8. Liu J, Cao R, Xu M, et al. Hydroxychloroquine, a less toxic derivative of chloroquine, is effective in inhibiting SARS-CoV-2 infection in vitro. Cell Discov. 2020;6:16. doi:10.1038/s41421-020-0156-0 
9. Sun X, Ni Y, Zhang M. Rheumotologitsts' view on the use of hydroxychloroquine to treat COVID-19. Emerg Microbes Infect. 2020;9(1):830-832. doi:10.1080/22221751.2020.1760145

10. Geleris J, Sun Y, Platt J, et al. Observational study of hydroxychloroquine in hospitalized patients with Covid-19. $N$ Engl J Med. 2020;382(25):2411-2418. doi:10.1056/NEJMoa2012410

11. Huang M, Li M, Xiao F, et al. Preliminary evidence from a multicenter prospective observational study of the safety and efficacy of chloroquine for the treatment of COVID-19. Natl Sci Rev. 2020;7(9):1428-1436. doi:10.1093/nsr/nwaa113

12. Mehra MR, Ruschitzka F, Patel AN. Retraction-Hydroxychloroquine or chloroquine with or without a macrolide for treatment of COVID-19: a multinational registry analysis. Lancet. 2020;395 (10240):1820. doi:10.1016/S0140-6736(20)31324-6

13. Rosenberg ES, Dufort EM, Udo T, et al. Association of treatment with hydroxychloroquine or azithromycin with in-hospital mortality in patients with COVID-19 in New York State. JAMA. 2020;323 (24):2493-2502. doi:10.1001/jama.2020.8630

14. Wang M, Cao R, Zhang L, et al. Remdesivir and chloroquine effectively inhibit the recently emerged novel coronavirus $(2019-\mathrm{nCoV})$ in vitro. Cell Res. 2020;30(3):269-271. doi:10.1038/s41422-020-0282-0

15. Hernandez AV, Roman YM, Pasupuleti V, Barboza JJ, White CM. Hydroxychloroquine or chloroquine for treatment or prophylaxis of COVID-19: a living systematic review. Ann Intern Med. 2020.

16. Lagier JC, Million M, Gautret P, et al. Outcomes of 3737 COVID-19 patients treated with hydroxychloroquine/azithromycin and other regimens in Marseille, France: a retrospective analysis. Travel Med Infect Dis. 2020;36:101791. doi:10.1016/j.tmaid.2020.101791

17. Risch HA. Early outpatient treatment of symptomatic, high-risk Covid-19 patients that should be ramped-up immediately as key to the pandemic crisis. Am J Epidemiol. 2020.

18. Zhou D, Dai SM, Tong Q. COVID-19 a recommendation to examine the effect of hydroxychloroquine in preventing infection and progression. J Antimicrob Chemother. 2020;75(7):1667-1670. doi:10. 1093/jac/dkaa114

19. Fantini J, Di Scala C, Chahinian H, Yahi N. Structural and molecular modelling studies reveal a new mechanism of action of chloroquine and hydroxychloroquine against SARS-CoV-2 infection. Int J Antimicrob Agents. 2020;55(5):105960. doi:10.1016/j.ijantimicag. 2020.105960

20. Podsiadlo P, Komiyama T, Fuller RS, Blum O. Furin inhibition by compounds of copper and zinc. J Biol Chem. 2004;279 (35):36219-36227. doi:10.1074/jbc.M400338200

21. Johnson BA, Xie X, Kalveram B, et al. Furin cleavage site is key to SARS-CoV-2 pathogenesis. bioRxiv. 2020.

22. Shittu MO, Afolami OI. Improving the efficacy of Chloroquine and Hydroxychloroquine against SARS-CoV-2 may require Zinc additives - a better synergy for future COVID-19 clinical trials. Infez Med. 2020;28(2):192-197.

23. Te Velthuis AJW, van den Worm SHE, Sims AC, Baric RS, Snijder EJ, van Hemert MJ. Zn2+ INHIBITS CORONAVIRUS AND ARTERIVIRUS RNA polymerase activity in vitro and zinc ionophores block the replication of these viruses in cell culture. PLoS Pathog. 2010;6(11):e1001176. doi:10.1371/journal.ppat.1001176

24. Carlucci P, Ahuja T, Petrilli CM, Rajagopalan H, Jones S, Rahimian J. Hydroxychloroquine and azithromycin plus zinc vs hydroxychloroquine and azithromycin alone: outcomes in hospitalized COVID-19 patients. medRxiv. 2020:2020.2005.2002.20080036.

25. Xiao L, Sakagami H, Miwa N. ACE2: the key molecule for understanding the pathophysiology of severe and critical conditions of COVID-19: demon or angel? Viruses. 2020;12:5. doi:10.3390/ v12050491
26. Puelles VG, Lütgehetmann M, Lindenmeyer MT, et al. Multiorgan and Renal Tropism of SARS-CoV-2. N Engl J Med. 2020;383 (6):590-592. doi:10.1056/NEJMc2011400

27. Butowt R, Bilinska K. SARS-CoV-2: olfaction, brain infection, and the urgent need for clinical samples allowing earlier virus detection. ACS Chem Neurosci. 2020;11(9):1200-1203. doi:10.102 1/acschemneuro.0c00172

28. Lenzer J. Covid-19: US gives emergency approval to hydroxychloroquine despite lack of evidence. BMJ. 2020;369:m1335. doi:10.1136/ bmj.m1335

29. COVID-19: chloroquine and hydroxychloroquine only to be used in clinical trials or emergency use programmes. Available from: https:// www.ema.europa.eu/en/documents/press-release/covid-19chloroquine-hydroxychloroquine-only-be-used-clinical-trialsemergency-use-programmes_en.pdf. Accessed October 9, 2020.

30. Magagnoli J, Narendran S, Pereira F, et al. Outcomes of hydroxychloroquine usage in United States Veterans Hospitalized with COVID-19. $\operatorname{Med}$ (N Y). 2020.

31. Prodromos CC. Hydroxychloroquine is protective to the heart, not Harmful: a systematic review. New Microbes New Infect. 2020;100747. doi:10.1016/j.nmni.2020.100747

32. Even those with "mild" symptoms can suffer from Covid-19 for a long time. Available from: https:/www.vrt.be/vrtnws/en/2020/06/ 19/even-those-wild-mild-symptoms-can-suffer-from-covid-19-for -a-lon/. 2020. Accessed October 9, 2020.

33. Arons MM, Hatfield KM, Reddy SC, et al. Presymptomatic SARS-CoV-2 infections and transmission in a skilled nursing facility. $N$ Engl J Med. 2020;382(22):2081-2090.

34. Gandhi M, Yokoe DS, Havlir DV. Asymptomatic transmission. The achilles' heel of current strategies to control Covid-19. N Engl J Med. 2020;382(22):2158-2160. doi:10.1056/NEJMe2009758

35. Lee SH, Son H, Peck KR. Can post-exposure prophylaxis for COVID-19 be considered as an outbreak response strategy in long-term care hospitals? Int J Antimicrob Agents. 2020;105988.

36. Jakhar D, Kaur I. Potential of chloroquine and hydroxychloroquine to treat COVID-19 causes fears of shortages among people with systemic lupus erythematosus. Nat Med. 2020;26(5):632. doi:10.1038/ s41591-020-0853-0

37. Chorin E, Dai M, Shulman E, et al. The QT interval in patients with COVID-19 treated with hydroxychloroquine and azithromycin. Nat Med. 2020;26(6):808-809. doi:10.1038/s41591-020-0888-2

38. Sacher F, Fauchier L, Boveda S, et al. Use of drugs with potential cardiac effect in the setting of SARS-CoV-2 infection. Arch Cardiovasc Dis. 2020;113(5):293-296. doi:10.1016/j.acvd.2020.04. 003

39. Hoffman T, Nissen K, Krambrich J, et al. Evaluation of a COVID-19 IgM and IgG rapid test; an efficient tool for assessment of past exposure to SARS-CoV-2. Infect Ecol Epidemiol. 2020;10(1): 1754538 .

40. Le Bert N, Tan AT, Kunasegaran K, et al. SARS-CoV-2-specific $\mathrm{T}$ cell immunity in cases of COVID-19 and SARS, and uninfected controls. Nature. 2020;584(7821):457-462. doi:10.1038/s41586-0202550-z

41. Wu F, Wang A, Liu M, et al. Neutralizing antibody responses to SARS-CoV-2 in a COVID-19 recovered patient cohort and their implications. medRxiv. 2020:2020.2003.2030.20047365.

42. Sekine T, Perez-Potti A, Rivera-Ballesteros O, et al. Robust T cell immunity in convalescent individuals with asymptomatic or mild COVID-19. Cell.

43. Wilson N, Kvalsvig A, Barnard LT, Baker M. Case-fatality risk estimates for COVID-19 calculated by using a lag time for fatality. Emergi Infect Dis J. 2020;26(6):1339. doi:10.3201/eid2606.200320 


\section{Publish your work in this journal}

Clinical Epidemiology is an international, peer-reviewed, open access, online journal focusing on disease and drug epidemiology, identification of risk factors and screening procedures to develop optimal preventative initiatives and programs. Specific topics include: diagnosis, prognosis, treatment, screening, prevention, risk factor modification,

Submit your manuscript here: https://www.dovepress.com/clinical-epidemiology-journal systematic reviews, risk \& safety of medical interventions, epidemiology \& biostatistical methods, and evaluation of guidelines, translational medicine, health policies \& economic evaluations. The manuscript management system is completely online and includes a very quick and fair peer-review system, which is all easy to use. 